\title{
Filigrane
}

Écoutes psychanalytiques

\section{Jeu, trauma complexe et travail d'élaboration psychique du jeu traumatique : illustration clinique}

\section{Miguel M. Terradas, Cécilanne Lepage-Voyer et Mélissa Paquette}

Volume 26, numéro 2, 2017

La terreur des enfants : deuxième partie

URI : https://id.erudit.org/iderudit/1055359ar

DOI : https://doi.org/10.7202/1055359ar

Aller au sommaire du numéro

Éditeur(s)

Revue Santé mentale au Québec

ISSN

1192-1412 (imprimé)

1911-4656 (numérique)

Découvrir la revue

Citer cet article

Terradas, M. M., Lepage-Voyer, C. \& Paquette, M. (2017). Jeu, trauma complexe et travail d'élaboration psychique du jeu traumatique : illustration clinique. Filigrane, 26(2), 45-60. https://doi.org/10.7202/1055359ar
Résumé de l'article

Le jeu est considéré par plusieurs auteurs comme étant le moyen privilégié utilisé par l'enfant pour mettre en scène, représenter et communiquer ses conflits intrapsychiques et ses difficultés relationnelles, de même que pour libérer ses fantasmes inconscients, et élaborer et moduler les angoisses liées à ces fantasmes. Les situations de négligence et de maltraitance au sein des premières relations significatives de l'enfant peuvent entraver le développement de sa capacité de symbolisation. Les abus physiques et sexuels vécus pendant l'enfance peuvent également provoquer une perte temporaire de la capacité de l'enfant à jouer et à faire semblant. Une autre conséquence est la présence de jeux et de thèmes traumatiques liés à la répétition littérale et compulsive des traumas. Cet article présente le travail de psychothérapie effectué auprès d'une jeune fille ayant vécu de multiples traumas avant l'âge de 3 ans. L'histoire de vie de l'enfant ainsi que sa situation de placement sont d'abord présentées. Ensuite, les différents jeux et thèmes symboliques et traumatiques sont illustrés à l'aide d'exemples cliniques. Les auteurs distinguent les jeux traumatiques des jeux abréactifs, ces derniers constituant une expression plus modérée de l'impact des traumas. Enfin, certaines attitudes et stratégies thérapeutiques visant l'élaboration psychique des traumas vécus par l'enfant sont discutées. 


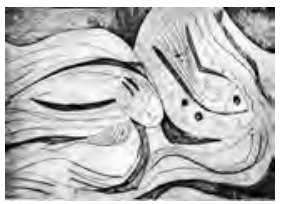

\title{
Jeu, trauma complexe et travail d'élaboration psychique du jeu traumatique: illustration clinique
}

\author{
Miguel M. Terradas, Cécilanne \\ Lepage-Voyer et Mélissa Paquette
}

RÉSUMÉ: Le jeu est considéré par plusieurs auteurs comme étant le moyen privilégié utilisé par l'enfant pour mettre en scène, représenter et communiquer ses conflits intrapsychiques et ses difficultés relationnelles, de même que pour libérer ses fantasmes inconscients, et élaborer et moduler les angoisses liées à ces fantasmes. Les situations de négligence et de maltraitance au sein des premières relations significatives de l'enfant peuvent entraver le développement de sa capacité de symbolisation. Les abus physiques et sexuels vécus pendant l'enfance peuvent également provoquer une perte temporaire de la capacité de l'enfant à jouer et à faire semblant. Une autre conséquence est la présence de jeux et de thèmes traumatiques liés à la répétition littérale et compulsive des traumas. Cet article présente le travail de psychothérapie effectué auprès d'une jeune fille ayant vécu de multiples traumas avant l'âge de 3 ans. L'histoire de vie de l'enfant ainsi que sa situation de placement sont d'abord présentées. Ensuite, les différents jeux et thèmes symboliques et traumatiques sont illustrés à l'aide d'exemples cliniques. Les auteurs distinguent les jeux traumatiques des jeux abréactifs, ces derniers constituant une expression plus modérée de l'impact des traumas. Enfin, certaines attitudes et stratégies thérapeutiques visant l'élaboration psychique des traumas vécus par l'enfant sont discutées. Mots-clés: jeu, trauma, jeu traumatique, attitudes et stratégies thérapeutiques, enfant

Abstract: Play is considered by several authors as the principal means used by the child to represent and communicate intrapsychic conflicts and relational difficulties, express unconscious fantasies, and elaborate and modulate anxieties related to these fantasies. Neglect and maltreatment within the early significant relationships may impair the development of the child's symbolic capacity. Physical and sexual abuse during childhood can also provoke a temporary loss of the child's ability for pretend play. Another consequence of maltreatment is the presence of posttraumatic themes and play activities related to a literal and compulsive repetition of traumas. This article presents the psychotherapy of a young girl who has been 
exposed to complex trauma before the age of 3. Firstly, the child's life history and different foster placements are recounted. Secondly, clinical examples are used to illustrate the symbolic and post-traumatic themes and play activities showed by the child in psychotherapy. The authors distinguish post-traumatic play segments from abreactive ones, the latter representing a moderate expression of the trauma. Finally, therapeutic attitudes and techniques aimed to facilitate the child's psychic elaboration of trauma are discussed.

Keywords: play, trauma, post-traumatic pay, therapeutic attitudes and techniques, child

\section{Introduction}

elon Alvarez (1988), le jeu est le moyen privilégié utilisé par l'enfant $\checkmark$ pour mettre en scène, représenter et communiquer ses conflits intrapsychiques et ses difficultés relationnelles, de même que pour libérer ses fantasmes inconscients. Il permet à l'enfant d'acquérir les bases nécessaires au développement de la communication interpersonnelle (Anzieu et Daymas, 2007), de la capacité à résoudre les problèmes (Chazan, 2002; Ferro, 2003) et de la régulation émotionnelle (Winnicott, 1939/1975). À travers le jeu, l'enfant découvre également de nouvelles habiletés qui renforcent son estime de soi et la maîtrise de son environnement (Kernberg et Normandin, 2000). Ces caractéristiques en font un outil clinique qui, d'une part, favorise la compréhension de l'organisation psychique, du niveau de développement (Chazan, 2002), des ressources et des difficultés de l'enfant, et qui, d'autre part, constitue une intervention thérapeutique appropriée pour ce groupe d'âge.

Cependant, le développement de la capacité à symboliser, jouer et faire semblant peut être grandement affecté lorsque l'enfant subit différentes formes de négligence ou de maltraitance. Les exemples suivants permettent d'illustrer ces propos. Premièrement, un enfant qui grandit dans un environnement suffisamment bon et qui développe de bonnes capacités à symboliser et à faire semblant peut présenter des difficultés temporaires à jouer lorsqu'il est affecté par un trauma isolé, tel qu'un abus sexuel extrafamilial. Dans ce cas, la psychothérapie vise d'abord à rétablir la capacité à jouer de l'enfant pour ensuite entamer le travail d'élaboration psychique du trauma (Terradas et Domon-Archambault, 2016). Deuxièmement, un enfant peut présenter des défaillances plus importantes au plan de ses capacités symboliques et de faire semblant lorsqu'il est exposé, de façon répétée et prolongée dans le temps, à de multiples événements traumatiques qui surviennent au sein de ses relations avec ses parents alors qu'il est en bas âge (Courtois, 
2004; Herman, 1992). Ces traumas prennent souvent la forme de comportements violents, de réponses inadaptées, imprévisibles ou inquiétantes de la part des parents (Bonneville, 2010; Courtois, 2004), ou d'abus physiques ou sexuels intrafamiliaux. L'enfant dépend encore totalement de ses parents, qui jouent un rôle fondamental dans le développement de sa capacité à percevoir et à élaborer les stimuli provenant de l'environnement. De plus, lorsque les parents sont impliqués dans des situations d'abus ou de maltraitance à l'égard de leur enfant, ils ne sont pas disponibles au plan psychique pour contenir le vécu affectif de ce dernier (Dubé, 2017). Les parents, submergés par leurs propres difficultés ou par un manque de patience et de tolérance, sont à la fois la source du trauma et les seuls individus vers lesquels l'enfant pourrait se tourner pour obtenir du réconfort en période de stress intense (Fonagy et Target, 1997). Selon Dubé, ce type de trauma met en péril la capacité de l'enfant à maintenir une proximité émotionnelle et un lien de confiance avec l'adulte. Le premier défi du psychothérapeute est donc d'établir une alliance de travail avec l'enfant. Il est ensuite nécessaire de lui permettre d'avoir accès au jeu, après quoi seulement la psychothérapie pourra commencer (Winnicott, 1971).

La présence de thèmes et de jeux traumatiques dans les activités ludiques de l'enfant est l'une des séquelles possibles des traumas vécus par ce dernier (Chazan, 2004; Romano, 2010; Terr, 1990, 1991). Les thèmes traumatiques (p. ex., torture, punition physique, mort) s'accompagnent fréquemment d'affects intenses d'anxiété, de tension, de désespoir ou de tristesse et, lorsque ces thèmes sont présents, le jeu peut prendre fin de façon catastrophique ou abrupte, sans résolution (Chazan, 2004). Pour sa part, le jeu traumatique se caractérise par la présence d'émotions négatives intenses pouvant devenir envahissantes et amener l'enfant à éviter ou à interrompre le jeu. Des thèmes liés au trauma sont mis en scène de manière rigide, répétitive et littérale (Terr, 1990, 1991). Selon Chazan et Cohen (2010), lors de la reviviscence d'un trauma, l'enfant, complètement dépassé par les événements, semble figé, déconnecté et tendu. Il peut également manifester de l'hypervigilance ou de la dissociation. Les pensées, les affects et les intentions qui sont exprimés dans le jeu sont fréquemment incohérents, et l'histoire qui l'accompagne est peu structurée (Chazan et Cohen, 2010).

Cet article vise à illustrer les différentes manifestations des jeux symboliques et traumatiques pouvant être observées chez un enfant ayant vécu de multiples traumas et à présenter quelques stratégies d'intervention soutenant le travail d'élaboration psychique de ceux-ci à travers le jeu. 


\section{Illustration et discussion d'un cas clinique : Myriam ${ }^{1}$}

Myriam avait 6 ans lorsqu'elle a été référée en psychothérapie dans l'objectif de l'aider à élaborer les conséquences des différents traumas vécus au sein de sa famille. L'enfant avait été victime de négligence extrême et d'abus physiques et sexuels durant les trois premières années de vie. Myriam aurait participé aux activités sexuelles de ses parents qui lui auraient été souvent présentées sous forme de jeu. Nous avons peu d'information concernant l'histoire de vie de l'enfant avant l'âge de 3 ans, moment où aurait eu lieu le signalement à la protection de la jeunesse qui a mené au placement de Myriam dans une famille d'accueil. La nature des abus a été connue plus tard, vers l'âge 5 ans, lorsque l'enfant a fait des verbalisations successives à sa mère d'accueil, provoquant chez cette dernière un trauma vicariant.

Lors des premières rencontres avec Myriam, le psychothérapeute a rapidement noté la présence de difficultés importantes au plan du langage expressif qui rendaient ardue la communication avec elle. Myriam avait été sous-stimulée au plan cognitif; elle manquait de mots pour s'exprimer et peinait à prononcer certains vocables. Heureusement, l'enfant persévérait dans ses tentatives de se faire comprendre et acceptait de répéter les phrases que le clinicien n'arrivait pas à décoder.

Malgré les multiples événements traumatiques vécus, Myriam avait réussi à développer une certaine capacité à faire du jeu symbolique. Nous émettons l'hypothèse selon laquelle la façon dont les abus sexuels se déroulaient, c'est-à-dire sous forme de jeu, lui aurait permis d'avoir accès au monde des fantaisies et des représentations. Bien que ces expériences soient aberrantes et insensées, elles auraient contribué au développement de l'imaginaire de Myriam, qui peut maintenant utiliser cette capacité pour mettre en scène les situations traumatiques, quelques fois sous forme de jeux traumatiques et, d'autres fois, à travers des jeux de faire semblant chargés de thèmes traumatiques. Ces observations concordent avec les résultats préliminaires d'une étude effectuée auprès des enfants québécois hébergés en contexte de protection de la jeunesse ayant vécu de multiples traumas ( $n$ $=15$ ). Selon cette recherche, le jeu de faire semblant et le jeu traumatique sont tous les deux présents, en alternance, chez la plupart des enfants évalués $(n=11)$ (Paquette et al., 2017).

Ainsi, dès les premières rencontres de psychothérapie, Myriam était capable de créer des scénarios imaginés et de jouer des personnages et des rôles variés. Ces habilités ont grandement contribué au travail d'élaboration psychique des traumas vécus par l'enfant au sein de sa famille d'origine. 
Nous relatons et discutons ici des principales phases du processus de psychothérapie de Myriam en prenant en compte les éléments suivants: le contexte de vie de l'enfant, ses difficultés et symptômes fondamentaux, les thèmes abordés en séance, les jeux mis en scène, ainsi que le transfert et le contre-transfert. Les diverses manifestations du jeu traumatique et certaines techniques de psychothérapie utilisées afin d'aider Myriam à élaborer les traumas sont illustrées. Pour ce faire, le processus thérapeutique est présenté en trois étapes. La première concerne le développement de l'alliance de travail et les premières manifestations du jeu traumatique. La deuxième relate l'évolution du jeu symbolique et l'élaboration des principaux thèmes traumatiques. La troisième illustre l'amorce du travail d'expression, de transformation et d'élaboration psychique des abus sexuels. Le processus thérapeutique étant en cours, certains thèmes nécessitent encore un travail de perlaboration.

\section{Étape 1 : Développement de l'alliance de travail et manifestations initiales du jeu traumatique}

Myriam habite en famille d'accueil depuis 3 ans. Elle ne voit plus ses parents biologiques, mais ils détiennent l'autorité parentale et doivent consentir aux interventions proposées à l'enfant. La famille d'accueil est composée du couple parental, de deux adolescentes issues de cette union, de Myriam et de sa sœur biologique, âgée de 3 ans, ayant été retirée de son milieu d'origine à la naissance. Au moment où la psychothérapie a débuté, la sœur de Myriam avait été légalement adoptée par la famille d'accueil.

Depuis un an, des souvenirs associés aux abus physiques et sexuels vécus émergent quasi quotidiennement chez l'enfant, qui les verbalise ou les manifeste à la mère d'accueil. Par exemple, Myriam utilise des mots vulgaires pour faire référence aux organes génitaux, elle a de la difficulté à respecter les limites corporelles de ses parents d'accueil et tend à toucher leurs parties intimes. De plus, elle se masturbe de façon compulsive, imite des activités sexuelles durant la nuit et peut introduire des objets dans son vagin. Étonnamment, elle rejette toute manifestation d'affection, verbale ou physique, provenant de la mère d'accueil. L'enfant a aussi initié des activités sexuelles avec sa sœur. La masturbation et les jeux sexualisés sont occasionnellement présents à l'école.

Myriam participe à une psychothérapie, à raison d'une séance hebdomadaire. Lors des rencontres, elle s'assoit spontanément quelques minutes et raconte au clinicien des événements de sa vie quotidienne. Durant la 
conversation, elle adopte une attitude séductrice à l'égard du psychothérapeute (p. ex., croise ses jambes comme une adulte, demande l'avis du psychothérapeute sur ses vêtements). Elle lui propose ensuite qu'ils jouent ensemble. Pourtant, durant cette période, elle l'implique peu dans ses activités ludiques. Myriam tolère bien la proximité physique du clinicien, tout en respectant les limites corporelles entre elle et lui. Suivant les idées de Romano (2010), trois types de jeu ont marqué cette étape du processus thérapeutique d'une durée approximative de huit mois, soit le jeu traumatique, le jeu abréactif et le jeu symbolique accompagné d'une réédition du jeu traumatique.

Illustration clinique d'un jeu traumatique. Premièrement, Myriam crée un scénario de jeu qui s'inspire du film La reine des neiges, mais qui en est très différent. La protagoniste, Elsa, une jeune fille qui habite avec ses parents, possède une belle chambre dans laquelle elle a un bureau, un ordinateur, une télévision et beaucoup de livres et de jouets. Myriam passe un long moment à organiser et à décorer cette chambre de «princesse » qui contraste avec le milieu carencé dans lequel elle a vécu avec ses parents biologiques. Elle introduit ensuite un nouveau personnage, qu'elle appelle Lona et qu'elle définit comme étant la meilleure amie d'Elsa. Lona est invitée à aller à la Ronde ${ }^{2}$ et à dormir chez son amie, mais elle doit d'abord demander la permission de ses parents. Le psychothérapeute est alors interpellé par Myriam pour jouer les personnages de la famille de Lona et représenter la scène où celle-ci demande la permission à ses parents pour aller chez son amie. Ensuite, Lona et ses parents doivent se présenter à la porte de la chambre d'Elsa où ils sont reçus par les parents de cette dernière.

Certains aspects sont apparus étranges au psychothérapeute et l'ont averti de la possible présence d'éléments traumatiques, soit le manque de considération de l'intimité et des frontières personnelles du personnage principal, ainsi que l'incongruence du contexte de jeu, représentée par le fait d'entrer dans une maison par la porte de la chambre de l'enfant. Ce dernier élément a été évoqué par Kernberg et Normandin (2000) en lien avec les caractéristiques du jeu d'enfants victimes d'abus sexuel (p. ex., manger dans la toilette, dormir dans le garage). Lorsque les parents de Lona partent, le jeu entre Elsa et son amie dure très peu de temps. Le psychothérapeute constate que Lona est plus intéressée aux parents d'Elsa qu'à son amie. Elle aide la mère d'Elsa dans la cuisine en même temps qu'elles «placotent» et rient ensemble, pendant qu'Elsa joue sur l'ordinateur dans sa chambre. Peu après, un jeu traumatique, d'une durée de quelques secondes, est mis 
en scène: Lona et la mère d'Elsa se collent et se frottent ensemble, l'une contre l'autre, pendant que Myriam simule des bisous. Quelques changements subtils sont ensuite observés dans la manière de jouer de Myriam, ce qui permet de supposer qu'elle est perturbée par le contenu du jeu mis en scène. Premièrement, dès le début de ce segment de jeu, l'enfant représente l'ensemble des personnages et ne demande plus au psychothérapeute de s'impliquer. Deuxièmement, elle ne donne aucune explication au clinicien concernant ses activités ludiques, ce qu'elle faisait pourtant auparavant. Troisièmement, l'enfant semble submergée par le jeu et ignore la présence du psychothérapeute, un comportement que Kernberg et al. (1998) nomment «solitaire conscient» pour qualifier les interactions sociales de certains enfants traumatisés. De façon similaire, Romano (2010) suggère que le jeu traumatique provoque une déliaison - au moins temporaire - des interactions avec le clinicien. En effet, rien n'est dit par Myriam qui, sous l'emprise du trauma, semble être dans un état pseudo-dissociatif ${ }^{3}$. Elle ne semble pas entendre les questions du clinicien visant à explorer le jeu mis en scène. Lors de cette reviviscence traumatique, Myriam reste figée et déconnectée de ce qui l'entoure, et répète ce scénario peu structuré qui se termine de façon abrupte lorsque le psychothérapeute annonce la fin de la séance. Ce jeu se présente, intact, lors des rencontres subséquentes.

Plusieurs attitudes et stratégies thérapeutiques ont été utilisées pour permettre à l'enfant d'élaborer, au moins partiellement, ce jeu traumatique. Elles sont présentées ici dans un certain ordre pour des raisons pédagogiques, mais elles ont été utilisées autant qu'il était nécessaire pour permettre à Myriam de se dégager de l'emprise du trauma et d'entreprendre le travail de symbolisation à travers le jeu. Premièrement, le clinicien a montré à Myriam qu'il était en mesure de contenir mentalement ses expériences traumatiques, en essayant de conserver la même attitude de neutralité bienveillante tout au long du processus thérapeutique. En effet, bien qu'il puisse être difficile de ne pas réagir au contenu traumatique, celui-ci doit être reçu et toléré par le psychothérapeute comme n'importe quel autre thème, afin d'éviter de perdre la capacité à penser ou d'avoir des réactions contretransférentielles extrêmes, telles que du déni ou de la fascination (Romano, 2010). Deuxièmement, comme suggéré par Kernberg et Normandin (2000), le clinicien a accepté que les traumas vécus par l'enfant étaient probablement impensables et indicibles, et que son histoire traumatique ne pouvait pas nécessairement être racontée. En ce sens, il a évité de poser des questions à Myriam concernant les traumas qu'elle avait subis. Lorsque possible, il lui 
posait des questions exploratoires visant à l'amener à expliquer davantage ce qui arrivait dans son jeu. Troisièmement, il a permis et toléré la répétition compulsive du jeu et des thèmes potentiellement associés au trauma, jusqu'à ce que la charge affective y étant liée diminue graduellement. C'est ainsi que l'enfant a réussi à se dégager partiellement des affects envahissants reliés au trauma pour ensuite gagner un certain contrôle sur ses activités ludiques (Terradas et Domon-Archambault, 2016). Terradas et DomonArchambault suggèrent plusieurs indices permettant d'identifier ces étapes cruciales du travail thérapeutique du jeu traumatique, soit l'arrêt partiel de la répétition compulsive du trauma, l'introduction par l'enfant d'autres éléments dans le contexte de jeu, l'établissement d'une certaine distance entre l'enfant et l'expérience traumatique et la reprise de la capacité narrative de l'enfant. Ils sont tributaires du degré de contrôle que l'enfant peut avoir dans la situation de jeu (Pynoss, 1994). Quatrièmement, afin de diminuer graduellement le recours à la dissociation chez Myriam, le psychothérapeute a fait plusieurs commentaires visant à souligner sa présence lors des séances. Progressivement introduits, ces commentaires ont permis à l'enfant de prendre conscience de la présence du clinicien et d'exprimer librement divers contenus traumatiques à travers le jeu.

Illustration clinique d'un jeu abréactif. Après plusieurs séances, Myriam introduit un nouveau personnage et complexifie le scénario de jeu. Mikael, le frère de Lona, joue avec les deux autres enfants et va à la Ronde avec elles. Sur la base des écrits de Romano (2010), cette évolution positive du jeu représenterait une tentative d'élaboration et d'assimilation du trauma sexuel, lui permettant de s'en dégager partiellement. Pourtant, des comportements, des pensées et des émotions associés à de possibles abus physiques font irruption dans le jeu: Mikael est victime de punitions excessives et injustifiées de la part de ses parents qui le réprimandent, l'isolent et le frappent souvent. Toutefois, durant ce segment de jeu, Myriam est en mesure d'entendre les questions du clinicien et d'y répondre, quoique de façon brusque et agressive, comme si elle répétait avec le psychothérapeute les comportements que ses parents auraient eus envers elle. Il est possible de penser que les abus physiques ont eu un impact moins néfaste sur la psyché de l'enfant que les abus sexuels; du moins, cette fois-ci, Myriam n'a pas recours à la dissociation pour faire face à la reviviscence du trauma. Elle semble s'identifier à l'agresseur, c'est-à-dire les parents de Lona et de Mikael, dans une tentative de regagner le contrôle sur des situations de maltraitance physique qu'elle aurait vécues passivement. 
Puisque le psychothérapeute n'est pas invité à participer au jeu, il commente les activités ludiques de l'enfant. Dans un premier temps, le travail interprétatif consiste fondamentalement à souligner l'agressivité injustifiée des parents à l'égard de Mikael. Dans un deuxième temps, le clinicien, identifié à la victime, met l'accent sur les sentiments qui peuvent habiter l'enfant lorsqu'il est la cible de l'agressivité des parents (p. ex., peur, tristesse, injustice, rage). Ces interventions ont favorisé une diminution graduelle des punitions à l'égard de Mikael après quelques rencontres.

Illustration clinique d'un jeu symbolique suivi d'une réédition d'un jeu traumatique. Myriam reconstruit la belle chambre d'Elsa dans laquelle il y a toujours - elle insiste - un bureau, un ordinateur et beaucoup de livres et de jouets. Elle introduit ensuite un nouveau personnage, «Anna», la petite sœur d'Elsa, un nom qu'elle emprunte à l'histoire de La reine des neiges. La chambre d'Anna, précise Myriam, n'est pas si belle et si grande que celle d'Elsa. L'enfant commence ensuite un nouveau jeu: Elsa est dans sa chambre lorsqu'Anna se fait attaquer dans le salon, situé à l'étage inférieur, par un monstre «qui veut la manger». Myriam exclame: «Le monstre lance du feu. Le feu entre dans son corps. Le monstre brûle son corps. Anna a le feu dans son corps.» Cette histoire donne lieu à un scénario dans lequel Anna est victime de l'attaque d'un monstre, représenté par une figurine masculine qui colle Anna et souffle le feu sur elle «comme un dragon». Elsa réussit à aider sa sœur à se libérer du monstre et à monter à sa chambre où elle se repose pendant qu'Elsa joue sur l'ordinateur. Plusieurs éléments importants sont exprimés à travers ce jeu symbolique. Premièrement, il est possible de penser que la rivalité entre Myriam et sa sœur, objet de sa jalousie depuis que cette dernière a été adoptée par leur famille d'accueil, se manifeste de deux façons. D'abord, par les différences entre les chambres des deux personnages, l'une très garnie et l'autre plutôt simple. Ensuite, par le fait que c'est Anna qui se fait agresser par le monstre. Ainsi, Myriam transforme défensivement la situation pour accorder certains avantages à Elsa, personnage avec lequel elle s'identifie. Ce mouvement défensif lui permet, d'une part, d'investir narcissiquement ce personnage afin de combler certains besoins d'amour et de réconfort, ici représentés par les biens matériels qui sont dans sa chambre et, d'autre part, de prendre une distance par rapport aux abus sexuels qu'elle a vécus, ce qui lui permet de gagner un certain contrôle sur la situation et ainsi éviter de répéter ses traumas de façon littérale et compulsive. Par ailleurs, la protagoniste joue sur l'ordinateur pendant que les autres personnages subissent des traumas. Nous émettons l'hypothèse selon laquelle 
Elsa, comme Myriam, recourt à la dissociation afin de se déconnecter des abus mis en scène dans le jeu. Bien que plusieurs interprétations relatives aux traumas sexuels puissent expliquer le contenu du jeu de l'enfant, le psychothérapeute n'a fait aucun commentaire ayant une valeur symbolique. Myriam n'était pas encore prête pour un travail d'élaboration des traumas, comme le démontre la réédition suivante du jeu traumatique. Quelques minutes plus tard, lorsqu'Anna se réveille, les deux sœurs jouent ensemble. Myriam entre dans un état pseudo-dissociatif, mais cette fois-ci moins intense, et ne donne aucune explication concernant ses activités ludiques. Les deux sœurs se collent et s'embrassent, l'une sur l'autre, dans le lit d'Elsa. Lorsque le psychothérapeute pose des questions sur le jeu entre les deux sœurs, l'enfant répète de façon automatique: «Elles jouent ensemble, elles jouent ensemble, elles jouent ensemble». Ici, Myriam apparait s'identifier à la fillette plus âgée, qui est l'agresseur, contrairement à ses jeux précédents, où elle s'identifiait à un enfant victime d'une femme adulte.

Cette première étape du processus de psychothérapie culmine avec une réflexion importante de Myriam concernant la nature abusive de la relation avec ses parents biologiques. L'enfant commente au début d'une séance: «Mes autres parents étaient méchants; mes nouveaux parents sont gentils avec moi. Je ne veux plus voir mes autres parents». Ce à quoi le clinicien a répondu: «Tes autres parents te faisaient des choses méchantes, des choses qu'on ne fait pas aux enfants». Puisque l'identification à l'agresseur faisait partie des mouvements défensifs de l'enfant, le psychothérapeute a pris la précaution de parler en termes de comportements inadéquats plutôt que d'insister sur l'expression "parents méchants», afin d'éviter que Myriam - qui provient de ces parents - puisse se sentir également méchante.

\section{Étape 2: Évolution du jeu symbolique et travail d'élaboration des principaux thèmes traumatiques}

Durant cette période de la psychothérapie, Myriam propose différents scénarios ludiques ayant en commun la présence de thèmes traumatiques, notamment des situations d'abandon, de négligence et de maltraitance. Les enfants, représentés par Les petits poneys empruntés des dessins animés, sont laissés à eux-mêmes, en l'absence des parents. Plusieurs situations sont mises en scène: les enfants manquent de nourriture, l'eau de la toilette déborde causant un gros dégât où «le pipi et le caca sont par terre», les enfants sont en danger et peuvent être kidnappés par un voleur. Le téléphone est toujours présent et joue un rôle central dans les activités ludiques 
de Myriam. Rainbow Dash, une «petite poney» représentée par l'enfant, est souvent malade. Elle a besoin de l'aide de son ami Flash, inspiré également du dessin animé, joué par le psychothérapeute. En l'absence des parents, les petits poneys doivent appeler l'ambulance, la police ou les pompiers pour les aider à faire face aux diverses situations adverses auxquelles ils sont confrontés. Les parents arrivent à la fin du jeu lorsque les enfants ont réussi à résoudre les différents problèmes. Ils sont parfois excessivement aimants et attentifs envers leurs enfants, d'autres fois démesurément sévères et punitifs. Les interprétations du clinicien abordent l'abandon des enfants et l'attitude ambivalente des parents.

Plusieurs éléments permettent de distinguer les histoires et les jeux élaborés par l'enfant pendant cette période de la psychothérapie de ceux qu'elle faisait auparavant. Premièrement, le clinicien est toujours invité à participer aux jeux de faire semblant et Myriam lui attribue différents rôles complémentaires aux siens. Cet élément évoque une évolution positive de l'alliance thérapeutique, permettant à l'enfant d'aborder les thèmes traumatiques sans recourir à la déliaison des interactions subjectives avec le clinicien (Romano, 2010) ou à la dissociation. Deuxièmement, elle est capable d'imaginer et de construire une histoire complexe et sensée dans laquelle s'inscrivent ses différentes activités ludiques, la continuité de la pensée (versus la discontinuité de la pensée; Berger, 2005) étant l'indicateur d'un certain progrès quant à l'élaboration des traumas. Troisièmement, elle est en mesure d'utiliser le jeu symbolique pour mettre en scène des situations potentiellement liées aux divers traumas qu'elle a vécus.

Un événement marquant se produit dans la vie de Myriam durant cette période du processus thérapeutique: troublée par les verbalisations à caractère sexuel de l'enfant, la famille d'accueil décide de mettre fin temporairement à leur engagement et demande le placement de Myriam. Quelques mois plus tard, il est décidé que l'enfant ne réintégrerait plus la famille d'accueil et qu'elle demeurerait au foyer de groupe où elle était alors hébergée. Les contacts avec la mère d'accueil ont été progressivement diminués.

Suite au déménagement au foyer de groupe, l'enfant propose le scénario suivant: la mère de Flash, représentée par Myriam, est très malade. Elle s'est évanouie à la maison et est amenée d'urgence à l'hôpital. Elle meurt dans son lit pendant que Flash doit crier: «Ma mère est malade, elle meurt! Je me sépare de ma mère; elle est très malade! Elle meurt!» Myriam joue dramatiquement le rôle de la mère de Flash, tout en dirigeant, pas à pas et avec précision, la mise en scène. Elle demande au psychothérapeute de crier de plus 
en plus fort la même phrase. Elle le corrige et lui montre comment le faire de façon encore plus dramatique. Ensuite, des policiers (personnages imaginés qui ne sont pas représentés ni par l'enfant ni par le psychothérapeute) obligent Flash à quitter l'hôpital de force, puisqu'il est interdit de crier. Le petit poney doit alors répéter en criant très fort: «Ma mère est morte! Je me sépare!» L'intensité des émotions exprimées par Flash témoignent de la profonde tristesse que Myriam semble vivre face à la séparation de la famille d'accueil. Ce scénario a grandement contribué au travail d'élaboration psychique du deuil relatif à la perte de cette famille. Ainsi, le déplacement des émotions ressenties face à la séparation de la mère d'accueil vers le clinicien - Flash - a permis à Myriam de se voir de l'extérieur et de regagner un certain contrôle sur la situation dans le contexte du jeu (Marvasti, 1994).

Pendant cette période, le clinicien constate que Myriam a besoin d'un moment de jeu calme dans la salle d'attente avant la séance de psychothérapie, afin d'être en mesure de tolérer le travail d'élaboration psychique des traumas mis en scène dans les jeux. En effet, lorsqu'elle arrivait tout juste avant le début de la séance, elle semblait en colère et n'acceptait pas les interventions du clinicien, préférant plutôt dessiner seule et refusant de partager le contenu de ses ouvres. Cette période de la psychothérapie a duré environ quatre mois.

\section{Étape 3: Travail d'expression, de transformation et d'élaboration psychique des abus sexuels}

Myriam s'est progressivement adaptée au foyer de groupe. Elle a fait des efforts importants pour surmonter ses difficultés d'apprentissage et obtenir de meilleurs résultats à l'école. Elle a appris, avec l'aide des éducateurs, à mieux réguler son comportement et à ne pas ventiler devant les autres enfants ou des étrangers les histoires des abus sexuels et physiques qu'elle aurait vécus. Les conduites masturbatoires ont grandement diminué et se présentent désormais uniquement dans sa chambre. Les comportements sexualisés envers les autres enfants ont presque disparu. Comme attendu au cours de l'évolution du processus de psychothérapie, l'intensification du transfert amène Myriam à mettre en scène des thèmes liés à la sexualité, sujet dont elle ne parle plus en dehors des rencontres.

Pendant plusieurs séances, Myriam propose un scénario dans lequel une «petite poney», Twilight, représentée par l'enfant, joue avec son ami Flash, toujours représenté par le psychothérapeute. Twilight «est une adolescente de 16 ans », précise Myriam; "elle mâche de la gomme et fait la fête avec ses 
amis». Elle a des pouvoirs magiques qui fascinent Flash. Le jeu mis en scène suit la séquence suivante: Twilight apprend à Flash à se battre avec les épées. Elle a un comportement séducteur à son égard et Flash est en admiration devant elle. Twilight devient agressive envers son ami alors que des pouvoirs très inégaux entre les personnages sont mis en scène. Elle attaque Flash sans compassion et rit sadiquement. Flash n'arrive pas à se défendre. Twilight coupe à répétition les membres de son corps pendant qu'elle lui dit des mots violents, humiliants et vulgaires. Myriam tire aussi des fléchettes sur le psychothérapeute; vers la fin des séances, elle vise ses parties intimes.

La nature des interactions ludiques entre Myriam et le clinicien évoque la notion de jeu abréactif proposée par Romano (2010) qui représente une tentative d'élaboration psychique du trauma. Ainsi, les pensées, les émotions et les sensations physiques exprimées par Myriam lors de cette séquence rappellent celles liées aux abus sexuels, mais elle réussit à s'en dégager partiellement en ayant recours inconsciemment au mécanisme d'identification à l'agresseur. Bien que la fin de cette scène soit jouée de manière littérale et compulsive, l'enfant trouve une solution partielle à son histoire. En s'identifiant à l'agresseur, elle transforme les violences qu'elle aurait subies en une situation où elle possède un certain contrôle (Kernberg et Normandin, 2000). De plus, malgré les émotions intenses qui habitent Myriam, caractérisées par un mélange de plaisir, de peur et d'angoisse, elle fait preuve d'autocontrôle et poursuit son jeu sans faire un passage à l'acte, en ce sens qu'elle ne touche jamais le clinicien, alors qu'elle exprime beaucoup d'agressivité envers Flash. Pour sa part, le psychothérapeute se devait de tolérer les sentiments contre-transférentiels générés par cette séquence ludique (un amalgame d'émotions liées aux rôles de victime - enfant abusé - et d'agresseur - adulte abusif), afin de montrer à l'enfant qu'il n'est pas détruit par les émotions qui lui sont projetées. Ses interventions visaient essentiellement à mettre en mots les états mentaux de la victime (p. ex., incompréhension, souffrance, manque de pouvoir, impuissance). Myriam écoute les interventions du clinicien, mais celles-ci ne semblent pas avoir un impact suffisant, puisque son comportement en contexte de jeu n'est pas modifié.

Une autre séquence ludique liée aux abus sexuels se présente lors d'une séance subséquente, alors que Myriam manifeste un intérêt pour les poupées sexuées. Twilight prend soin de sa petite sœur et de son petit frère, représentés par les poupées. Elle leur donne à manger, les déshabille, les lave, change leurs couches et leurs vêtements. Peu après, Myriam, surexcitée et anxieuse, se cache pour simuler des activités sexuelles entre les jeunes enfants. Son 
comportement devient de plus en plus désorganisé. En effet, l'enfant transgresse les limites et s'approche du psychothérapeute de façon envahissante dans le but de l'impliquer dans ses activités ludiques. À ce moment, elle n'est plus dans un jeu de faire semblant, mais plutôt dans une répétition littérale et compulsive des traumas sexuels. Des pulsions partielles, orales (p. ex., imiter des fellations entre les poupées), anales, urétrales (p. ex., faire caca et pipi sur autrui) et phalliques (p. ex., caresser les organes génitaux des poupées) se manifestent par des comportements désorganisés où se mélangent le plaisir associé à la surexcitation, l'anxiété, la peur et un certain dégoût. Myriam insiste pour que Flash participe à ces «jeux», ce que le psychothérapeute refuse de faire en évoquant la nature de la relation entre les personnages (frères et sœurs), l'interdit de l'inceste et le caractère abusif de la scène. Identifiée à l'adulte-abusif, Myriam fait vivre des émotions contre-transférentielles intenses au clinicien qui, identifié à l'enfant-abusé, perd temporairement sa capacité de penser. Par la suite, le psychothérapeute décide de rester dans le scénario proposé par l'enfant. C'est ainsi que Flash évoque la nécessité d'avoir la présence bienveillante d'un adulte qui empêcherait les enfants de se livrer à ces pulsions partielles. L'enfant est aux prises avec un amalgame d'émotions, alors qu'elle semble d'une part frustrée de ne pas pouvoir élaborer ses pulsions sexuelles et agressives dans son jeu et, d'autre part, soulagée, le psychothérapeute ayant été en mesure de la protéger de ses propres pulsions.

Préoccupé par l'intensité des affects exprimés par l'enfant et la nature des rapports transférentiels et contre-transférentiels, le clinicien revient sur le contenu du jeu à la fin de la rencontre pour souligner, tout en faisant allusion aux personnages, le caractère abusif de la situation. Il insiste sur l'effet que l'absence d'un adulte bienveillant peut avoir sur les comportements des enfants. Étant laissés à eux-mêmes, ils ne sont pas en mesure de savoir ce qui est permis ou interdit au plan sexuel.

\section{Conclusion}

La psychothérapie de Myriam étant inachevée, il est difficile de proposer une juste conclusion. Il est cependant possible de souligner deux éléments pouvant être essentiels au travail d'élaboration psychique des traumas vécus par l'enfant. En termes d'attitude thérapeutique, la présence mentale du clinicien, caractérisée par son ouverture d'esprit et sa tolérance, a permis à l'enfant de transformer, au moins partiellement, ses angoisses les plus profondes à travers le jeu (Blake, 2008). En termes de stratégie d'intervention, 
il a été crucial pour l'évolution de l'enfant de rester dans le jeu (Kernberg et Normandin, 2000). C'est uniquement dans ce contexte que l'enfant s'est permise d'exprimer les sentiments les plus terrifiants, sans peur de conséquences. Ainsi, Myriam a pu symboliquement agresser, humilier, séduire et avoir peur du psychothérapeute, tout en constatant qu'il gardait une attitude bienveillante à son égard. Enfin, cet article souligne la pertinence du jeu comme outil thérapeutique auprès des enfants ayant vécu des traumas.

\section{Miguel Terradas \\ Miguel.Terradas.Carrandi@USherbrooke.ca Mélissa Paquette Cécilanne Lepage-Voyer}

\section{Notes}

1. Prénom fictif, afin de préserver l'anonymat de l'enfant.

2. La Ronde est un grand parc d'attraction avec des manèges pour enfants et adultes situé à Montréal.

3. Les auteurs préferent utiliser le terme «pseudo-dissociation" pour faire référence à une rupture temporaire, de très courte durée, de fonctions mentales qui sont habituellement intégrées. Dans ce cas, il s'agit de la conscience de ses propres affects et de la perception de l'environnement (présence du clinicien).

\section{Références}

Alvarez, A. (1988). Beyond the unpleasure principle: Some preconditions for thinking through play. Journal of Child Psychotherapy, 14, 1-13.

Anzieu, A. et Daymas, S. (2007). Introduction. Dans A. Anzieu, C. Anzieu-Premmereur et S. Daymas (dir.), Le jeu en psychothérapie de l'enfant (p. 3-11). Paris, France: Dunod.

Berger, M. (2005). L'enfant instable. Approche clinique et thérapeutique (2 éd.). Paris: Dunod. Blake, P. (2008). The setting, physical and mental, and limits. Dans P. Blake. Child and adolescent psychotherapy (p. 193-208). Londres: Karnac.

Bonneville, E. (2010). Effets des traumatismes relationnels précoces chez l'enfant. La psychiatrie de l'enfant, 53, 31-70. doi: 10.3917/psye.531.0031

Chazan, S. E. (2002). Profiles of Play: Assessing and Observing Structure and Process in Play Therapy. Londres: Jessica Kingsley Publishers.

Chazan, S. E. (2004). Children's Play Therapy Instrument (CPTI). Manual (version révisée par S. E. Chazan du Children's Play Therapy Instrument (CPTI), Kernberg, P. F., Chazan, S. E., et Normandin, L. (1997)). New York. Document inédit.

Chazan, S. E. et Cohen, E. (2010). Adaptative and defensive strategies in post-traumatic play of young children exposed to violent attacks. Journal of Child Psychotherapy, 36 (2), 133-151.

Courtois, C. A. (2004). Complex trauma, complex reactions: Assessment and treatment. Psychotherapy: Theory, Reasearch, Practice, Training, 41 (2), 412-425.

Dubé, G. (2017). Mentalisation, empathie et comportements extériorisés chez les enfants hébergés en centre jeunesse. Université de Sherbrooke, Canada. Thèse de doctorat inédite. 
Ferro, A. (2003). Le jeu. Dans A. Ferro. L'enfant et le psychanalyste (p. 81-107). Paris: Érès.

Fonagy, P. et Target, M. (1997). Attachment and reflective function: their role in self-organization. Development and Psychopathology, 9, 679-700. doi : 10.1017/S0954579497001399

Herman, J. L. (1992). Complex PTSD: A syndrome in survivors of prolonged and repeated trauma. Journal of Traumatic Stress, 5 (3), 377-391.

Kernberg, P. F., Chazan, S. E. et Normandin, L. (1998). The Children's Play Therapy Instrument (CPTI). Description, Development, and Reliability studies. The Journal of Psychotherapy Practice and Research, 7 (3), 196-207.

Kernberg, P. F. et Normandin, L. (2000). Children Play Therapy Instrument adapted for sexually abused children. Unpublished manuscript, Cornell Medical School, New York Presbyterian Hospital, New York.

Marvasti, J. A. (1994). Please hurt me again: Posttraumatic play therapy with an abuse child. Dans T. Kottman et C. Shaefer (dir.), Play Therapy in action: A casebook for practitioners (p. 485-525). New Jersey: Jason Aronson.

Pynoos, R. (1994). Traumatic stress and developmental psychopathology in children and adolescents. Dans R. Pynoos (dir.), Posttraumatic stress disorder. A clinical review (p. 65-98). Baltimore: The Sidran.

Paquette, M., Terradas, M. M., Chazan, S. E. et Lepage, C. (soumis). Les représentations d'attachement, les relations d'objet et le jeu des enfants victimes de mauvais traitements. Bulletin de psychologie.

Romano, H. (2010). Traces du trauma dans les «jeux» d'enfants victimes d'évènements traumatiques. Le journal des psychologues, 279, 57-61.

Terr, L. (1990). Too scared to cry: Psychic trauma in childhood. New York: Harper \& Row Publishers.

Terr, L. C. (1991). Childhood traumas: An outline and overview. The American Journal of Psychiatry, 148 (1), 10-20.

Terradas, M. M. et Domon-Archambault, V. (2016). Conceptualisation de la psychothérapie d'enfants à la lumière des défis rencontrés en pédopsychiatrie et en protection de l'enfance. La psychiatrie de l'enfant, 59 (2), 537-574.

Winnicott, D. W. (1971). Jeu et réalité: L'espace potentiel. Paris: Gallimard.

Winnicott, D. W. (1975). Pourquoi les enfants jouent-ils? Dans Winnicott, D. W. L'enfant et le monde extérieur (p. 123-128). Paris: Payot. Travail original publié en 1939. 